\title{
Socially induced diet preference can partially reverse a LiCl-induced diet aversion
}

\author{
BENNETT G. GALEF, JR. \\ McMaster University, Hamilton, Ontario, Canada
}

\begin{abstract}
I examined the capacity of a socially induced enhanced diet preference to reverse the effects of a LiCl-induced diet aversion. I found that rats poisoned after eating a novel diet (Diet NPT) would consume substantial amounts of Diet NPT following interaction with a conspecific that had eaten Diet NPT. Neither rats interacting with a conspecific fed some other diet nor rats exposed to Diet NPT itself exhibited reduced aversion to Diet NPT. This surprising capacity of social interaction to ameliorate even profound toxicosis-induced aversions suggests that social influence may be a major experiential determinant of the diet preferences of free-living rats.
\end{abstract}

In the vast majority of studies of diet preference, diet selection is observed in subjects maintained and tested in isolation from one another. Such isolation of subjects, although it ensures independent measures of preference across subjects, precludes detection of social influences on diet selection. In consequence, a possibly important experiential determinant of diet selection by free-living animals has largely been ignored in laboratory studies of determinants of food choice.

Recent experiments conducted both in our laboratory and elsewhere indicate that social interactions among Norway rats can substantially alter the diet selection of naive rats faced with a choice between novel foods (Galef $\&$ Wigmore, 1983; Posadas-Andrews \& Roper, 1983; Strupp \& Levitsky, 1984). A naive rat (an observer) that encounters a recently fed conspecific (a demonstrator) an hour or two after that demonstrator has eaten will, for many hours thereafter, exhibit a marked increase in its preference for the diet eaten by its demonstrator (Galef, 1983; Galef \& Kennett, 1985; Galef \& Wigmore, 1983; Strupp \& Levitsky, 1984).

A number of studies have demonstrated that when social influence is the sole independent variable in an experiment, it produces robust alterations in diet acceptance (e.g., Galef, Kennett, \& Wigmore, 1984). However, such experiments have not provided information on the relative importance of social influences on diet preference when such influences act within the context of other determinants of diet selection (e.g., learned aversions, diet familiarity, etc.). There have been no data indicating the magnitude of socially induced changes in diet preference relative to other experiential determinants of diet selec-

This research was supported by grants from both the Natural Sciences and Engineering Research Council of Canada and McMaster University Research Board. I thank Cecilia Malinski for technical assistance and the faculty and staff of the Department of Psychology, University of Colorado at Boulder, for their hospitality and provision of resources during the drafting of this paper. Requests for reprints should be sent to Bennett G. Galef. Jr., Department of Psychology, McMaster University, Hamilton, Ontario L8S-4K1, Canada. tion, and there has been no understanding of the ways in which social influence might interact with other factors affecting diet acceptance.

In the experiments described below, I examined the extent to which socially induced enhanced diet acceptance could ameliorate a diet aversion induced by $\mathrm{LiCl}$ toxicosis. My goal was to provide a rough indication of the magnitude of the effects of social interaction on acceptance of a diet relative to poison-induced aversions to the same diet. If it should be the case that social interactions induce negligible alterations in toxicosis-based diet aversions, then one might be justified in ignoring social factors in discussions of the ontogeny of diet selection. If, on the contrary, social influences markedly enhanced intake of a diet avoided because of its previous association with a toxin, social influence would have to be considered an important factor within the complex of determinants of diet selection.

\section{GENERAL METHOD}

\section{Subjects}

Experimentally naive 42-day-old rats, obtained from Blue Spruce Farms (Altamont, NY), served as observers. The demonstrators were 9- and 10-week-old rats that had been used as observers in previous experiments.

\footnotetext{
Apparatus

The subjects were housed and tested as demonstrator-observer pairs in $42.5 \times 24 \times 27.5 \mathrm{~cm}$ wire-mesh hanging cages (Wahmann Co., Baltimore, MD). Each hanging cage was divided into two equal parts by a $1.25-\mathrm{cm}(1 / 2-i n$.) screen partition $(24 \times$ $27.5 \mathrm{~cm}$ ) attached at the midpoints of the $42.5-\mathrm{cm}$ sides of each cage.

\section{Procedure}

Treatment of subjects during experiments was as follows (see Figure 1):

Step 1. Demonstrator and observer were maintained together with ad-lib access to Purina Laboratory Chow pellets for a 1-day period of familiarization with both enclosure and pair-mate.

Step 2. The demonstrator was moved to the opposite side of the enclosure and was fed ad lib for another $24 \mathrm{~h}$, while the observer was food deprived in anticipation of induction of a learned taste aversion.
} 

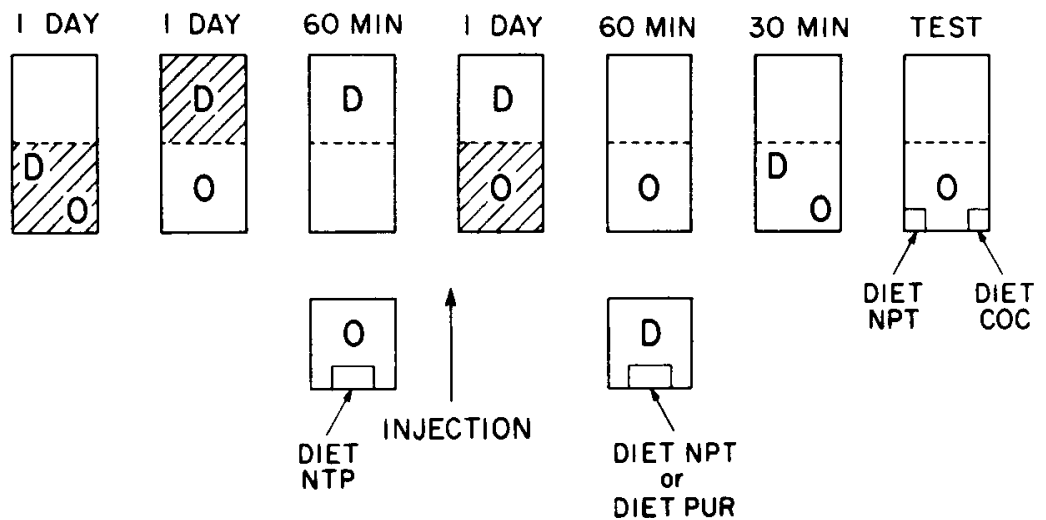

Step: I

2

3

4

5

6

7

Figure 1. Schematic diagram of the general procedure.

Step 3. The observer was moved to an enclosure in a separate room and was fed, for $60 \mathrm{~min}$, a casein- and sucrose-based diet, Diet NPT (Normal Protein Test Diet, Catalogue No. 170590, Teklad Diets, Madison, WI). Immediately after the feeding period, each bowl of Diet NPT was weighed, and each observer received an intraperitoneal injection of solution.

Step 4. After injection, the observer was returned to its home cage and was fed Purina Laboratory Rodent Chow for $24 \mathrm{~h}$. During this 24-h period, each demonstrator was food deprived so that it would eat when given the opportunity to do so (Step 5).

Step 5. The demonstrator was moved to an enclosure in a separate room and was fed, for $60 \mathrm{~min}$, either Diet NPT (see Step 3) or Diet PUR (powdered Purina Laboratory Rodent Chow). During this 60-min period the food was removed from the observer's side of the cage in anticipation of Steps 6 and 7.

Step 6. The demonstrator was introduced into the observer's side of the cage and demonstrator and observer were allowed to interact freely for $30 \mathrm{~min}$.

Step 7. The demonstrator was removed from the experiment and the observer was offered, for $22 \mathrm{~h}$, a choice between weighed samples of two diets, Diet NPT and Diet COC (powered Purina Laboratory Rodent Chow adulterated $2 \%$ by weight with Hershey's Pure Cocoa). At the end of the 22-h test period, the experimenter again weighed the samples of diet, determined the amount of each diet eaten, and calculated the percentage of Diet NPT eaten by each observer.

\section{Data Analysis}

The percentage of Diet NPT eaten by observers that interacted with demonstrators fed Diet NPT and Diet PUR was compared using the Mann-Whitney $U$ test, two-tailed. A p of less than .05 was considered statistically significant.

\section{EXPERIMENT 1}

Experiment 1 was undertaken to determine the extent to which interaction with a demonstator fed Diet NPT would ameliorate an aversion to Diet NPT previously acquired by an observer.

\section{Method}

Subjects. One hundred and twenty 42-day-old Long-Evans rat pups served as observers; 96 9- or 10-week-old rats served as demonstrators. Data from 4 observers were discarded because their demonstrators failed to eat during Step 5 of the experiment.
Procedure. The procedure was as described under General Method (see Figure 1). During Step 3 of the experiment, three groups, each composed of 24 observers, were injected with $1 \%$, $.5 \%$, or $.25 \%$ of body weight, respectively, $\mathrm{LiCl}$ solution $(1 \% \mathrm{w} / \mathrm{v})$.

Forty-eight additional observers were assigned to two control conditions. The 24 subjects assigned to the saline control group were injected with $.5 \%$ of body weight saline solution during Step 3 of the experiment. The 24 observers in the exposure control group were injected with $.5 \%$ of body weight, $1 \%(w / v) \mathrm{LiCl}$ solution in Step 3. During Step 6, the observers in the exposure control group interacted for $30 \mathrm{~min}$, not with a demonstrator fed Diet NPT or Diet PUR, but with a food bowl containing either Diet NPT $(n=12)$ or Diet PUR $(n=12)$.

The ideal design of the present experiment would have involved nine groups, rather than the five I examined. There should have been saline and exposure control groups at all three levels of toxicosis induction, not just one. Practical constraints prohibited the conducting of a fully balanced experiment and I examined only those gruops of subjects I felt were most likely to provide useful information.

\section{Results}

The main results of Experiment 1 are presented in the left-hand panel of Figure 2, which shows the mean amount of Diet NPT eaten, as a percentage of total amount ingested, by observers during the 22 -h test period. As can be seen in Figure 2, interaction with a demonstrator that had eaten Diet NPT had the capacity to partially ameliorate the effects of a learned aversion to that diet. This amelioration of a toxicosis-induced aversion to Diet NPT is most clearly seen in the diet selection of the experimental group of observers that were administered $.5 \%$ of body weight $\mathrm{LiCl}$ solution after eating Diet NPT and prior to exposure to a demonstrator that had eaten Diet NPT.

During the 22-h test period, mean total intake of Diets NPT and COC by individual observers in the five groups ranged from 19.7 to $23.3 \mathrm{~g}$. Thus, the changes in percentage intake of Diet NPT shown in Figure 2 represent substantial enhancement of amount of Diet NPT eaten by observers exposed to demonstrators fed Diet NPT.

Comparison of the behavior of observers in the saline 
EXPERIMENT I

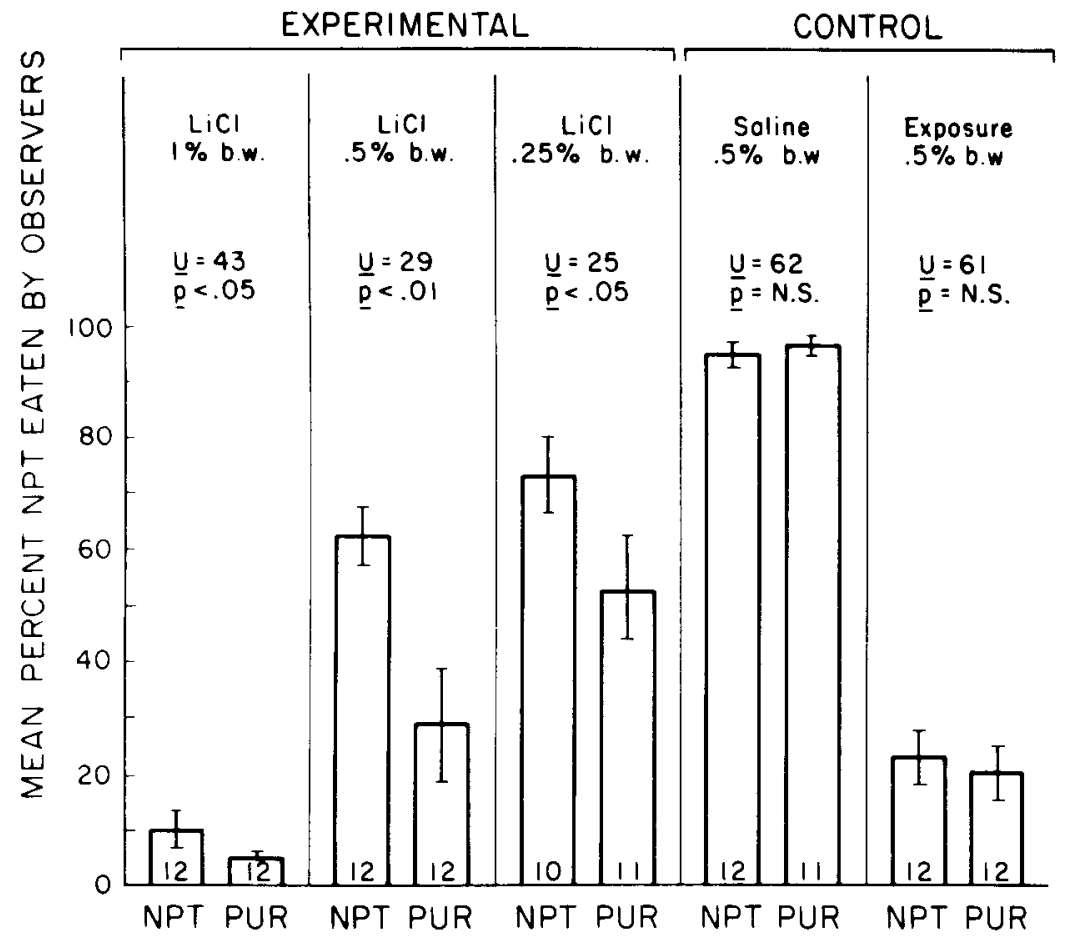

EXPERIMENT 2

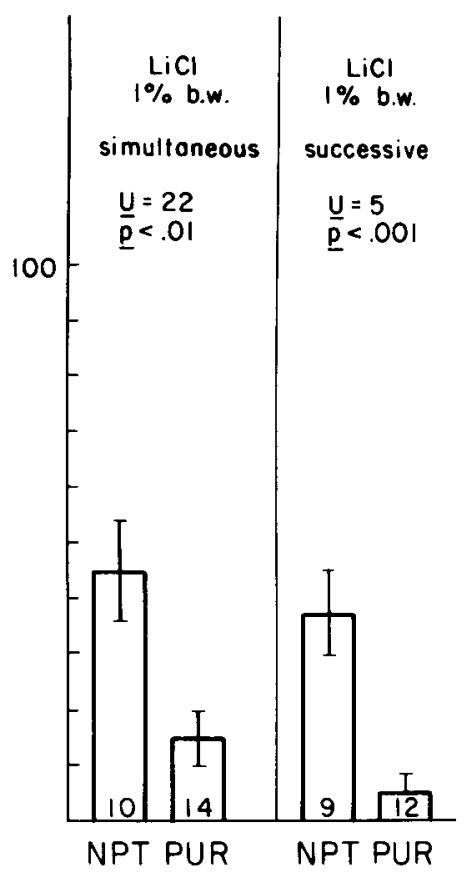

Figure 2. Mean percentage of Diet NPT eaten by observers during $22 \mathrm{~h}$ of testing. See Method sections of Experiments 1 and 2 for explanation of groups. Numbers within histograms $=$ N/group. Flags indicate +1 SEM.

control group with that of observers injected with $.5 \%$ of body weight of $\mathrm{LiCl}$ solution reveals that even with a moderate level of toxicosis induction, the influence of a demonstrator only partially reversed an observer's aversion to Diet NPT. Observers that were injected with .5\% of body weight $\mathrm{LiCl}$ solution and interacted with a demonstrator fed Diet NPT ate a significantly smaller percentage of Diet NPT during testing than observers that were injected with saline solution and interacted with demonstrators fed Diet NPT (Mann-Whitney U test, $\mathrm{U}=1$, $\mathrm{p}<.001)$.

Examination of the data from the group of observers in which a profound aversion to Diet NPT was induced (those observers injected with $1 \%$ of body weight of $1 \%$ $\mathrm{LiCl}$ solution) reveals that although interacting with a demonstrator fed Diet NPT significantly enhanced intake of that diet, the magnitude of the effect on the acceptance of Diet NPT was trivial.

Finally, as we have shown previously (Galef, Kennett, \& Stein, 1985; Galef \& Stein, 1985), examination of the diet selection of observers in the exposure control condition (those that interacted during Step 6 with food bowls of Diets NPT and PUR, rather than those with demonstrators fed those diets) indicates that it was social interaction with fed demonstrators, rather than simple exposure to a food, which was critical in ameliorating the aversion to Diet NPT exhibited by subjects in the various experimental conditions. Observers that interacted with a demonstrator fed Diet NPT during Step 6 ingested a significantly greater percentage of Diet NPT during the 22- $\mathrm{h}$ test than did those observers interacting with a bowl of Diet NPT during Step 6 (Mann-Whitney U test, U = $22, \mathrm{p}<.01)$.

In general, the results of Experiment 1 indicate that interaction for 30 min with a demonstrator fed Diet NPT can partially reverse a mild $\mathrm{LiCl}$-induced aversion to that diet, but that a profound aversion to Diet NPT is relatively unaffected by such interaction.

\section{EXPERIMENT 2}

Experiment 2 was undertaken to determine whether interaction of an observer with more than one demonstrator (fed the diet that an observer had learned to avoid) could substantially ameliorate a profound aversion to the avoided diet.

\section{Method}

Subjects. Forty-eight 42-day-old Long-Evans rat pups served as observers and 96 9- or 10-week-old rats served as demonstrators.

Procedure. The procedure was essentially that described under General Method, with two exceptions: First, demonstrators were introduced into the experimental apparatus only during Step 6 of the experiment (see Figure 1). Second, each of the 48 observers, following injection with $1 \%$ of body weight, $1 \% \mathrm{w} / \mathrm{v} \mathrm{LiCl}$ solution, interacted with 2 demonstrators during Step 6 of the procedure. Each of 24 observers interacted for $30 \mathrm{~min}$ with a pair of demonstrators, both of which had been fed either Diet PUR or Diet NPT during Step 5 of the procedure, that were introduced simultaneously into the apparatus during Step 6. The remaining 24 observers interacted for $15 \mathrm{~min}$ with each of 2 demonstrators, both of which had been fed either Diet PUR or Diet NPT during Step 5, 
that were introduced into the experimental apparatus in succession during Step 6.

\section{Results and Discussion}

As can be seen in the right-hand panel of Figure 2, which shows the amount of Diet NPT eaten by observers as a percentage of total amount ingested during the 22-h test period, interaction with 2 demonstrators, presented either sequentially or simultaneously, substantially ameliorated a profound $\mathrm{LiCl}$-induced aversion to Diet NPT.

\section{GENERAL DISCUSSION}

Observers in Experiment 1 interacted with a single, familiar demonstrator; observers in Experiment 2 interacted with 2 unfamiliar demonstrators. This confounding of demonstrator number and familiarity makes it impossible to unequivocally attribute the difference in Diet NPT acceptance seen in observers in Experiments 1 and 2 injected with $1 \%$ of body weight $\mathrm{LiCl}$ solution to the difference in the number of demonstrators they encountered during Step 6 of the procedure.

However, the results of a previous study that directly examined the effects of demonstrator familiarity on transmission of diet preference from demonstrators to observers revealed no effect of demonstrator familiarity on magnitude of social influence (Galef, Kennett, \& Wigmore, 1984, Experiment 3). It is, therefore, likely that the increased size of the effect of demonstrators on observers seen in Experiment 2, as compared with Experiment 1 , is the result of the greater number of demonstrators inducing intake of Diet NPT in Experiment 2. This issue obviusly needs to be examined directly.

In any case, the results of Experiment 2, unlike those of Experiment 1, indicate that exposure to demonstrators fed Diet NPT can substantially ameliorate a profound LiCl-induced aversion to Diet NPT.
Taken together, the results of Experiments 1 and 2 suggest that socially induced diet preferences are of sufficient relative magnitude to play an important role in the diet selection of free-living animals. Although social influence on diet preference does not appear to be as robust as the influence of toxicosis, social influence can substantially reduce the magnitude of toxicosis-induced aversions. This capacity of social interactions to counter the effects of learned flavor aversions, the most potent of known experiential determinants of diet selection, is far greater than I would have anticipated on the basis of the results of previous studies of social influence on diet selection.

\section{REFERENCES}

GALEF, B. G., JR. (1983). Utilization by Norway rats ( $R$. norvegicus) of multiple messages concerning distant foods. Journal of Comparative Psychology, 97, 364-371.

GAlef, B. G., JR., \& KENNetT, D. J. (1985). Delays after eating: Effects on transmission of diet preferences and aversions. Animal Learning \& Behavior, 13, 39-43.

Galef, B. G., JR., Kennett, D. J., \& Stein, M. (1985). Demonstrator influence on observer diet preference: Effects of simple exposure and the presence of a demonstrator. Animal Learning \& Behavior, 13, $25-30$.

Galef, B. G., JR., KennetT, D. J., \& Wigmore, S. W. (1984). Transfer of information concerning distant food in rats: A robust phenomenon. Animal Learning \& Behavior, 12, 292-296.

GALEF, B. G., JR., \& SteIN, M. (1985). Demonstrator influence on observer diet preference: Analyses of critical social interactions and olfactory signals. Animal Learning \& Behavior, 13, 31-38.

GaleF, B. G., JR., \& Wigmore, S. W. (1983). Transfer of information concerning distant foods: A laboratory investigation of the 'information-centre' hypothesis. Animal Behaviour, 31, 748-758.

Posadas-Andrews, A., \& Roper, T. J. (1983). Social transmission of food preferences in adult rats. Animal Behaviour, 31, 265-271.

Strupp, B. J., \& Levitsky, D. A. (1984). Social transmission of food preferences in adult hooded rats (Rattus norvegicus). Journal of Comparative Psychology, 98, 257-266.

(Manuscript received April 29, 1985; revision accepted for publication October 28, 1985.) 